\title{
Bilateral Plasticity of Vibrissae SII Representation Induced by Classical Conditioning in Mice
}

\author{
Weronika Debowska, ${ }^{1}$ Monika Liguz-Lecznar, ${ }^{1}$ and Malgorzata Kossut ${ }^{1,2}$ \\ ${ }^{1}$ Department of Molecular and Cellular Neurobiology, Nencki Institute, 02-093, Warsaw, Poland, and ${ }^{2}$ Warsaw School of Social Sciences and Humanities, \\ 03-815, Warsaw, Poland
}

The somatosensory cortex in mice contains primary (SI) and secondary (SII) areas, differing in somatotopic precision, topographic organization, and function. The role of SII in somatosensory processing is still poorly understood. SII is activated bilaterally during attentional tasks and is considered to play a role in tactile memory and sensorimotor integration. We measured the plasticity of SII activation after associative learning based on classical conditioning, in which unilateral stimulation of one row of vibrissae was paired with a tail shock. The training consisted of three daily $10 \mathrm{~min}$ sessions, during which 40 pairings were delivered. Cortical activation driven by stimulation of vibrissae was mapped with $2-\left[{ }^{14} \mathrm{C}\right]$ deoxyglucose $(2 \mathrm{DG})$ autoradiography $1 \mathrm{~d}$ after the end of conditioning. We reported previously that the conditioning procedure resulted in unilateral enlargement of 2DG-labeled cortical representation of the "trained" row of vibrissae in SI. Here, we measured the width and intensity of the labeled region in SII. We found that both measured parameters in SII increased bilaterally. The increase was observed in cortical layers II/III and IV. Apparently, plasticity in SII is not a simple reflection of changes in SI. It may be attributable to bilateral integrative role of SII, its lesser topographical specificity, and strong involvement in attentional processing.

\section{Introduction}

The second somatosensory representation (SII) in the cerebral cortex was first described in cats by Adrian (1941). In mice, the localization of SII was discovered by Woolsey (1967) and the area was mapped by Carvell and Simons (1987). SII contains the entire body representation in a somatotopic map, but receptive fields are larger than in primary somatosensory area (Burton and Carlson, 1986; Carvell and Simons, 1987). It is reciprocally connected with the ipsilateral somatosensory (SI) and motor cortex (Carvell and Simons, 1987; Miyashita et al., 1994), ventral posterolateral (VPL), ventral posteromedial (VPM), and posterior (PO) nucleus of the thalamus (Burton and Kopf, 1984; Theyel et al., 2010), and the prefrontal cortex (Kostopoulos et al., 2007). SII sends projections to the premotor cortex (Rizzolatti and Luppino, 2001), the striatum, and to insular and perirhinal cortices (Shi and Cassell, 1998). Callosal connections link SII with contralateral SI and SII (Carvell and Simons, 1987). Facial vibrissae, which are prominently represented in SI, occupy $\sim 14 \%$ of SII (Carvell and Simons, 1986). SII was considered to be dependent on SI input (Bohlhalter et al., 2002), but recent data in rodents, derived from studies of direct thalamic input and of activation of SII in the absence of SI-SII connection, show that the concept of hierarchically organized pathway no longer holds (Theyel et al., 2010). It is

Received Nov. 15, 2010; accepted Dec. 15, 2010.

This work was supported by statutable funds of the Nencki Institute. We thank Dr. Ewa Siucinska for help with initial experimentation.

Correspondence should be addressed to Prof. Malgorzata Kossut, Department of Molecular and Cellular Neurobiology, Nencki Institute, Pasteur 3, 02-093, Warsaw, Poland. E-mail: kossut@nencki.gov.pl.

DOI:10.1523/JNEUROSCI.5989-10.2011

Copyright $\odot 2011$ the authors $\quad 0270-6474 / 11 / 315447-07 \$ 15.00 / 0$ more likely that SII processes sensory input also in parallel with SI.

In this context, it was relevant to examine experiencedependent plasticity in SII. Use-dependent plasticity of functional representations in SI is very well documented, but only little work on SII has been done, with the notable exception of an early paper by Pons et al. (1988). In cats, burst stimulation of skin results in potentiation of field potentials and increased firing rates of SII units (Manzoni et al., 1979). Involvement of SII in learning was suggested in macaque monkeys, where tool use learning was found to be associated with increased gray matter in SII in both hemispheres (Quallo et al., 2009). In humans, tactile coactivation of many receptive fields on a finger induced increase in blood oxygen level-dependent signal in contralateral and ipsilateral SI and SII (Pleger et al., 2003). An important recent paper by Sacco and Sacchetti (2010) suggests a role of secondary sensory cortices in emotional memory.

The vibrissae-to-cortical barrels system of rodents, a very popular model for studies of cortical use-dependent plasticity, is well suited for examining SII plasticity, since the vibrissal representation there is well identified. We reported previously that short-lasting classical conditioning, in which unilateral whisker stimulation is paired with tail shock, results in expansion of functional representation of the row of vibrissae activated during the training, in contralateral SI. Here, we report that, after the same conditioning paradigm, vibrissal representation, visualized with $2-\left[{ }^{14} \mathrm{C}\right]$ deoxyglucose (2DG) autoradiography, is enlarged in SII of both hemispheres. We also reexamined the lateralization of input from the vibrissae in control and conditioned animals. 


\section{Materials and Methods}

\section{Animals and behavioral training}

We used 28 adult (7-8 weeks of age during conditioning) female C57BL/6J mice. Mice were kept in a temperature-controlled room $\left(20^{\circ} \mathrm{C}\right)$ with a natural light/dark cycle. They had ad libitum access to water and food. To habituate to experimental conditions, all animals were accustomed to a neck restraint by being placed in a custom-built restraining apparatus for $10 \mathrm{~min}$ a day for 3 weeks before the start of experiments. All experimental procedures on living animals were approved by First Warsaw Local Ethical Commission for Animal Experimentation Commission and were in accordance with the European Communities Council Directive of 24 November 1986.

Conditioned group (conditioned stimulus plus unconditioned stimulus). Mice $(n=12)$ were placed in a neck restraining apparatus, and row B of vibrissae, on one side of the muzzle, was stimulated manually with a fine paint brush. The stimulation lasted $9 \mathrm{~s}$ and consisted of three strokes; each stroke lasted $3 \mathrm{~s}$ [conditioned stimulus (CS)]. During the last second of the last stroke, a single tail shock [unconditioned stimulus (UCS)] was applied $(0.5 \mathrm{~s}, 0.5 \mathrm{~mA})$. After a $6 \mathrm{~s}$ interval, the stimuli were repeated. The training consisted of three daily 10 min sessions, during which 40 pairings were delivered.

Pseudoconditioned group (pseudo). Animals in this group $(n=6)$ received the same number of stimuli per session as the mice in the conditioned group, but stimuli were unpaired. Stimulation of a row B was applied for the same duration; tail shock was given at random relative to whisker stimulation.

Control group (control). Mice in this group $(n=10)$ were not subjected to the training procedure but only habituated to the neck restraint.

\section{2-Deoxyglucose mapping}

2DG mapping was performed $24 \mathrm{~h}$ after the end of training or after the habituation period in control mice. The mice received a single intramuscular dose of 2- $\left[{ }^{14} \mathrm{C}\right]$ deoxy-D-glucose $(5 \mu \mathrm{Ci} /$ mouse; American Radiolabeled Chemicals; specific activity, $55 \mathrm{mCi} / \mathrm{mmol}$ ).

Stimulation of vibrissae was bilateral or unilateral. In the groups with bilateral stimulation (CS+UCS, $n=6$; pseudo, $n=6$; control, $n=5$ ), rows $\mathrm{B}$ vibrissae on both sides of the snout were stroked with $2 \mathrm{~Hz}$ frequency, in a rostrocaudal direction, using a mechanical stimulator, for 45 min (all whiskers except rows B were trimmed 10 min before the 2DG injection). In the groups with unilateral stimulation, row B vibrissae used during conditioning (CS+UCS; $n=5)$ or in control group $(n=5)$, row $B$ vibrissae on the left or right side of the muzzle were stimulated, whereas whiskers of the other rows were clipped close to the skin. The whiskers on the other side of the muzzle were left intact. The mice were then anesthetized $(0.2 \mathrm{ml} / \mathrm{mouse})$ with Vetbutal (Biowet) and briefly perfused with $4 \%$ paraformaldehyde in phosphate buffer $(0.1 \mathrm{~m}), \mathrm{pH}$ 7.4. The brains were removed; hemispheres were dissected and flattened between glass slides, and then frozen with isopentane $\left(-70^{\circ} \mathrm{C}\right)$ and sectioned $(30 \mu \mathrm{m}$ thick) in a plane tangential to the barrel cortex using a cryostat $\left(-18^{\circ} \mathrm{C}\right)$. The serial sections were collected onto glass slides, briefly dried on a hot plate $\left(+60^{\circ} \mathrm{C}\right)$ to prevent the diffusion of the radioactive marker, and then placed against mammography x-ray film (MIN-R 2000; Kodak) together with radioactivity ${ }^{14} \mathrm{C}$ standards (GE Healthcare; American Radiolabeled Chemicals; specific activity, $53 \mathrm{mCi} / \mathrm{mmol}$ ) for 2 weeks. After obtaining the autoradiograms, the sections were Nissl-stained to identify the brain structures.

\section{Analysis of autoradiograms}

The autoradiograms were analyzed using a computer image analysis system (MCID 4; Imaging Research). The barrels were identified on the basis of Nissl-stained sections. SII was identified basing on the surface maps of Carvell and Simons (1986). Labeling was quantified as described by Siucinska and Kossut (1996). The criterion for labeling was that the intensity of 2DG uptake should be at least $15 \%$ higher than in the surrounding cortex. All image files were coded so that the experimenter did not know the identity of the sample.

We measured the width of the band of increased 2DG uptake in the secondary somatosensory cortex (in layers II-III and layer IV) and in the barrel cortex (in layer IV) (see Fig. $1 B, C$ ). We also measured the intensity of labeling (2DG uptake) in layers II-III and IV. The intensity of 2DG labeling within SII, which represents global neuronal activity in this region, was always normalized to the background intensity in nearby region of cortex to exclude individual differences related to metabolism in the 2DG uptake. The results are expressed in arbitrary units, as the ratio of average SII labeling to the average background labeling. For analysis of ipsilateral band of labeling in SII, area of the site of higher 2DG uptake was measured and intensity of labeling in relation to the barrel field was estimated. All measurements were performed on three sections per layer for each hemisphere. The values obtained were averaged for all sections from each layer.

Statistical analysis was performed using the GraphPad Prism 5 software (GraphPad Software). Comparisons were made using one-way ANOVA with Tukey-Kramer multiple-comparison test. Interhemispheric comparisons of the 2DG labeling were made with two-tailed $t$ test.

\section{Videorecording}

To acquire a behavioral evidence that conditioning occurred, mice were videorecorded during all three sessions. Trials during which a mouse moved its head and reacted vigorously in response to stimulation of vibrissae were considered in the analysis. Only head movements after CS application were counted. The results were calculated using the twotailed $t$ test.

\section{Results}

\section{Behavioral analysis}

In the conditioned group, we observed a lowering of behavioral reaction, expressed as head movements in response to CS. The observed reduction in head movements is akin to freezing observed during fear conditioning in which footshock is used as UCS (Fanselow and Poulos, 2005) and can be used as an indicator of learning (Cybulska-Klosowicz et al., 2009).

In the first pairing session, the mice showed a head movement after $28 \pm 7.8 \%$ of CS presentations, and in the third session, after $13 \pm 3.7 \%$. The difference in head movement scores between the sessions was statistically significant $(p<0.05)$. In the course of training, no significant differences in behavioral reaction was found in the pseudoconditioned group $(30 \pm 4.7 \%$ during first session; $23.5 \pm 7.4 \%$ in third session), which is consistent with our previous reports (Jasinska et al., 2010).

\section{DG autoradiography}

\section{Bilateral stimulation}

Bilateral stimulation of row B vibrissae produced in each hemisphere an accumulation of label visible as two bands, one in the barrel cortex and another, shorter band, ventrally from the barrel cortex (Fig. 1 $A, B$ ). This ventral band corresponded to location of SII (Carvell and Simons, 1986). The two regions were clearly separated in layers II-IV but were less distinct in infragranular layers.

Width of row B representation in SII. Analysis of the autoradiograms showed a significant effect of the type of sensory training on a representation of the row B in secondary somatosensory cortex. Additional comparison revealed that after the conditioning, in layer IV row B representation in the SII was wider than in the pseudoconditioned and in the control group by 32 and $33 \%$, respectively (ANOVA, $F_{(2,13)}=14.2 ; p=0.0005$ ), in the hemisphere contralateral to row of whiskers stimulated during conditioning ("trained"); and by 12 and $18 \%\left(F_{(2,13)}=13.71 ; p=\right.$ 0.0006 ) in the ipsilateral hemisphere (Figs. $2,3 B$ ). In layer II-III, the width of row B representation in SII of the CS+UCS group was by 38 and $42 \%$ greater on the contralateral side $\left(F_{(2,12)}=\right.$ 8.036; $p=0.0061)$ and by 20 and $29 \%\left(F_{(2,14)}=7.731 ; p=\right.$ 

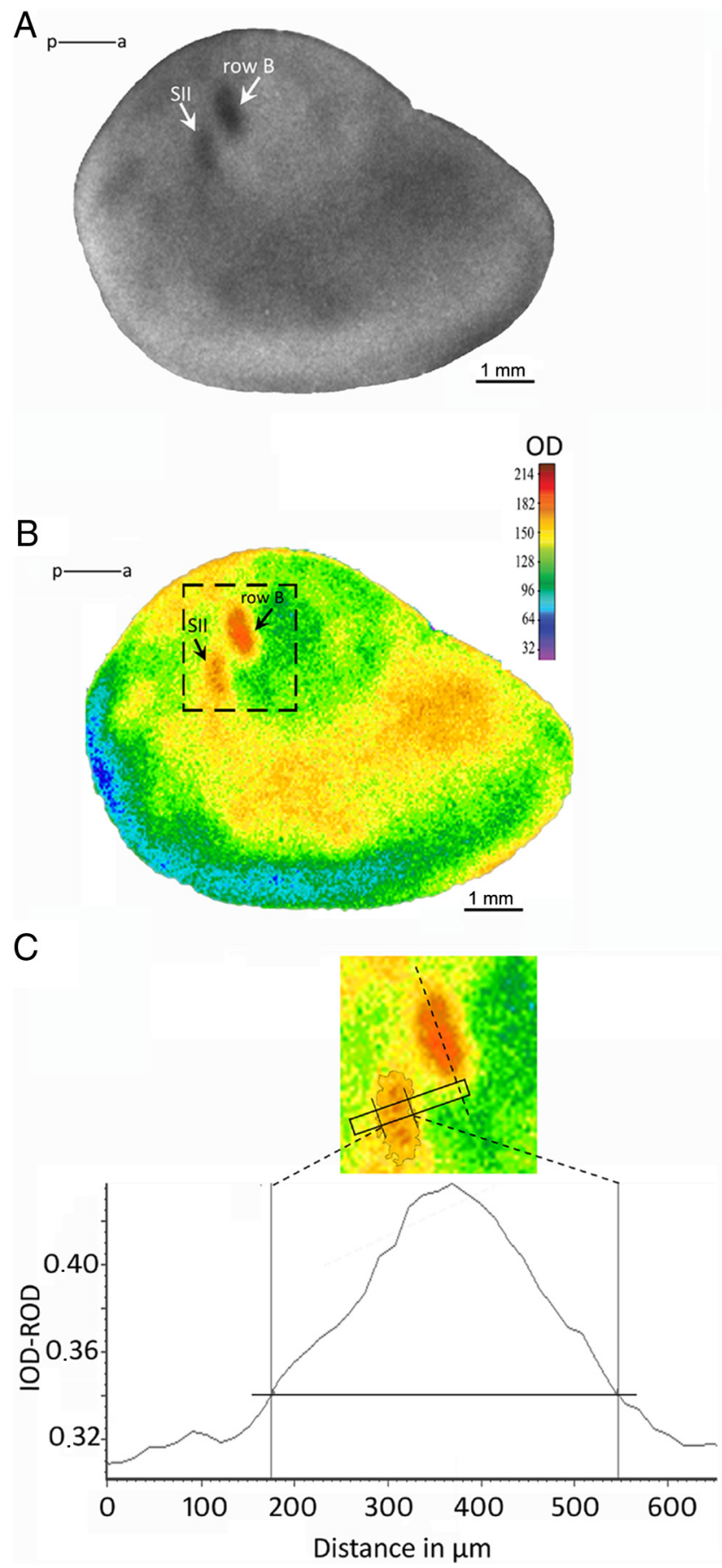

Figure 1. Neuronal activity mapped with $2 D G$ autoradiography. $A$, A typical autoradiogram of tangential section through layer IV, showing increased 2DG incorporation in primary (SI) and secondary (SII) somatosensory areas after sensory stimulation of row B whiskers. $\boldsymbol{B}$, The same autoradiogram in pseudocolors. The most active regions are represented in red, and the less active, in blue. C, Scheme of measurements. The diagram shows the plot of optical density across the marked region of SII (rectangle). The region for measurements in SII is placed perpendicularly to the long axis of the stimulated row representation in SI (dashed line). The horizontal line indicates 2DG labeling 15\% higher than the mean background level. IOD-ROD, Optical density in arbitrary units; $\mathrm{p}-\mathrm{a}$, posterior-anterior axis.

0.0055 ) on the ipsilateral side than in pseudo and control groups, respectively (Figs. 2, 3A).

In SI, in accordance with our previous findings (Siucinska and Kossut, 1996), the analysis showed a significant enlargement of the representation of the trained row in the barrel cortex, by $25 \%$
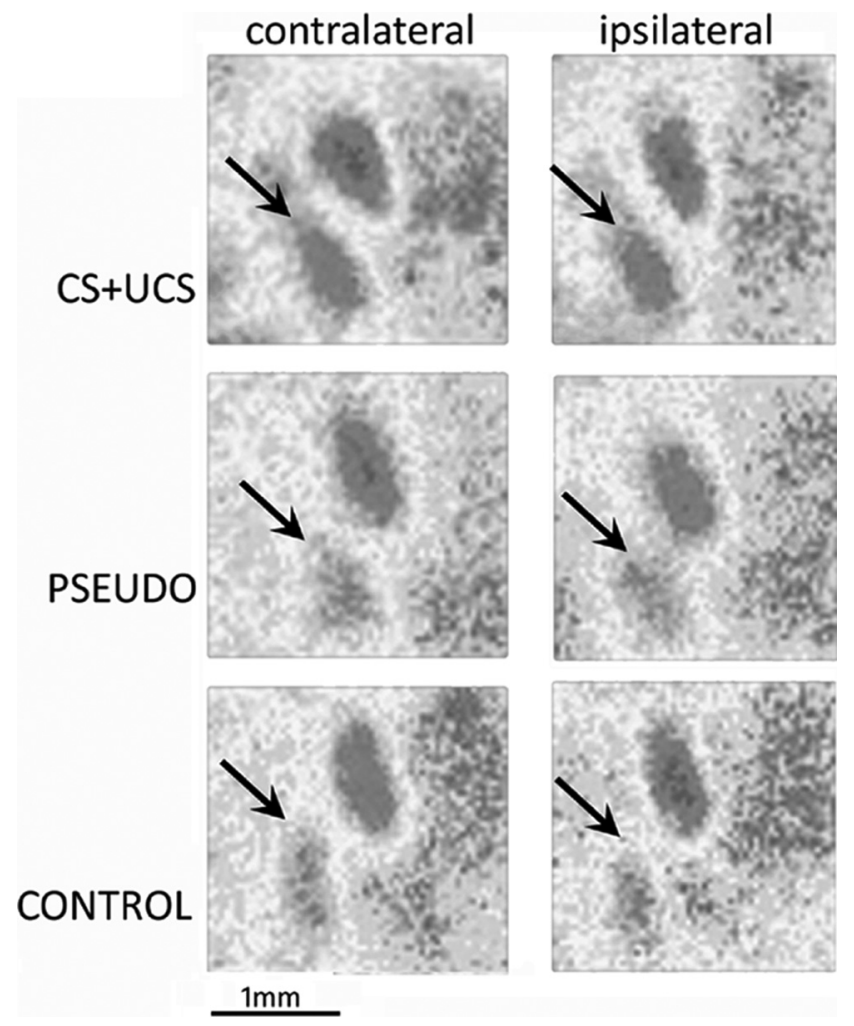

Figure 2. 2DG autoradiograms showing the effect of associative sensory training on func tional plasticity of primary and secondary somatosensory cortices. Pictures represent tangential sections across the cortex at the level of layer IV. Pairing of conditioned sensory stimulus with an unconditioned aversive stimulus (CS + UCS) results in unilateral widening of the stimulated row representation in SI and bilateral widening of SII (marked with arrow) compared with control and pseudoconditioned groups. Additionally, in SIl, sensory training entails a bilateral increase of 2DG labeling intensity.

$(457.7 \pm 9.6 \mu \mathrm{m}$ in trained vs $366.5 \pm 6.4 \mu \mathrm{m}$ in ipsilateral hemisphere; two-tailed $t$ test, $p<0.005$ ) (Fig. 2).

Intensity of labeling. In layer IV of the hemisphere contralateral to the trained vibrissae, intensity of labeling in SII was higher in CS+UCS group than in both pseudoconditioned and control groups. We also found a statistically significant difference between the pseudoconditioned and the control group $\left(F_{(2,13)}=\right.$ 21.93; $p<0.0001)$. We observed the same effect in the ipsilateral hemisphere $\left(F_{(2,13)}=40.78 ; p<0.0001\right)$ (Figs. 2, 3D). In layer II-III, we found significant changes only between CS+UCS group and the two other groups, in both the contralateral $\left(F_{(2,13)}=\right.$ 37.23; $p<0.0001)$ and ipsilateral $\left(F_{(2,13)}=22.88 ; p<0.0001\right)$ hemispheres (Figs. 2, 3C).

\section{Unilateral stimulation}

Unilateral stimulation of row B vibrissae in control mice did not evoke any focused increase in 2DG uptake in the ipsilateral barrel field and SII (Fig. 4). In mice that underwent classical conditioning, 2DG uptake to stimulation of the trained row $\mathrm{B}$, examined $24 \mathrm{~h}$ after the training, revealed a patch of higher 2DG uptake (Figs. 4, 5) near ipsilateral barrel field. The patch was irregular in shape, most often elongated, with long axis arranged in parallel with SII and located medial and posterior to row A barrels. It was partly overlapping (in its anterior part) with labeling of SII evoked by stimulation of contralateral row B barrels. Comparing with labeling evoked in SII by stimulation of contralateral row of vibrissae, the intensity of $2 \mathrm{DG}$ labeling of the patch was $\sim 20 \%$ lower and the width of labeling was $45 \%$ shorter. 

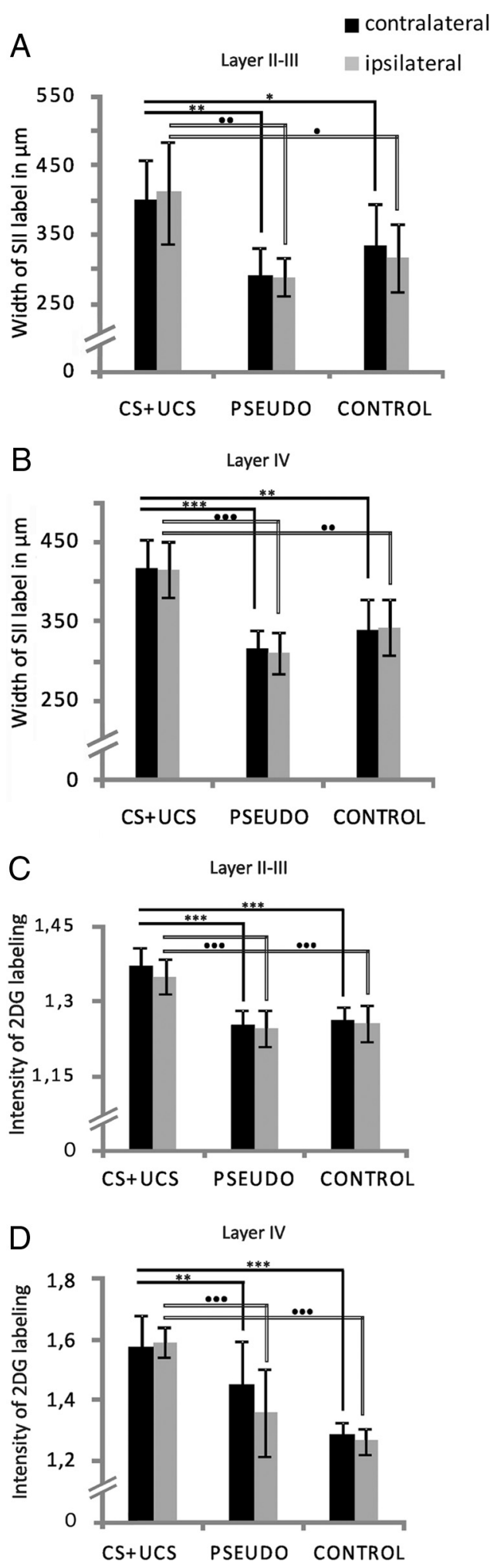

Figure 3. Quantification of training-induced changes in width $(\boldsymbol{A}, \boldsymbol{B})$ and intensity $(\boldsymbol{C}, \boldsymbol{D})$ of SII labeling in cortical layers II-III $(\boldsymbol{A}, \boldsymbol{C})$ and IV $(\boldsymbol{B}, \boldsymbol{D})$. In both analyzed layers, the width and the intensity of $2 D G$ labeling were always significantly greater in trained animals than in pseudoconditioned or control animals. These increases occurred in both hemispheres. * Comparison between groups for hemisphere contralateral to the stimulated row of whiskers; ${ }^{\bullet}$ comparison for hemisphere ipsilateral to the stimulated row of whiskers. ${ }^{*} \bullet_{p}<0.05$; ${ }^{* *}, \bullet_{p}<0.01$; ${ }_{* * *}, 0_{p}<0.001$. Intensity of labeling is expressed in arbitrary units. Error bars represent SD.

\section{Discussion}

The important finding of this study is that plasticity of SII is not a simple reflection of plasticity in adjacent, ipsilateral SI. During posttraining 2DG mapping of brain activation, when the trained row of whiskers on one side of the snout and control row on the other side were stimulated, enlargement of activated regions was observed in both ipsilateral and contralateral SII fields, and in SI contralateral to the trained row. When posttraining unilateral stimulation of the trained row was applied, ipsilateral SII showed a patch of activation that was not present in control mice. Thus, classical conditioning, in which one row of whiskers was involved, modified the response of SII cortex to vibrissal input bilaterally, whereas in SI this plastic change was unilateral. This is the first demonstration of bilateral modification of sensory representations in the SII cortex arising as a result of conditioning.

SII integrates somatosensory information from both sides of the body (Carvell and Simons, 1986, 1987; Iwamura, 2000). The SII fields are connected by callosal fibers, and their role may not be simply combining excitatory signals of two hemispheres to yield bilateral receptive fields. Experiments using denervation or reversible inactivation have attributed transcallosal activity a modulatory role in bilateral interactions (Shuler et al., 2001). In addition to the direct callosal projection, SII and SI fields in the two hemispheres can also interact via fibers descending to the thalamus (Raij et al., 2008). Experiments by Li and Ebner (2006) have shown that activity in the barrel cortex on one side can influence thalamic relay neurons on the contralateral side. In that way, interhemispheric integration can interact with corticothalamic coordination to modulate cortical activity. Therefore, the sensory vibrissal input directed primarily to contralateral SI can indirectly modulate the responsiveness of ipsilateral SII.

The question of parallel versus hierarchical information processing in SI and SII has been addressed by several recent studies. In rats, independent inputs to SI and SII were described with anatomical and electrophysiological techniques (Kwegyir-Afful and Keller, 2004), and a direct parallel pathway to SII from VPL was demonstrated (Liao and Yen, 2008). A recent study in mice using flavoprotein autofluorescence imaging in brain slices (Theyel et al., 2010), combined with anatomical and electrophysiological experiments confirmed it. In fact, SII neurons receive as much, if not more, thalamic input as they do from SI (BrettGreen et al., 2003), at least in the rat. Moreover, there is a proportion of thalamic neurons that has double projections to SI and SII, which suggests parallel processing (Liao and Yen, 2008). Theyel et al. (2010) found that connections between SI and SII are not necessary to activate SII, but activation of SI can reach SII via posterior nucleus of the thalamus. This partial independence of SII may be one of the reason for explaining the differences in the plasticity of cortical representations in the two somatosensory areas. And yet, the increased contralateral SII response to stimulation of trained row of vibrissae was not transmitted to ipsilateral SII. Instead, a small band of activation, situated partly outside the region normally activated by row B vibrissae stimulation, was visible. To reveal not only that patch of activation but also enlargement of what can be regarded as regular whisker representation, as observed in control animals, it was necessary to stimulate the control row of vibrissae, on the other side on the muzzle. Apparently, activation of this learning-induced modification of SII vibrissal representation relies on priming by the principal, contralateral sensory input.

The plastic changes in extent and intensity of SII activation were observed in both hemispheres, contralateral and ipsilateral 

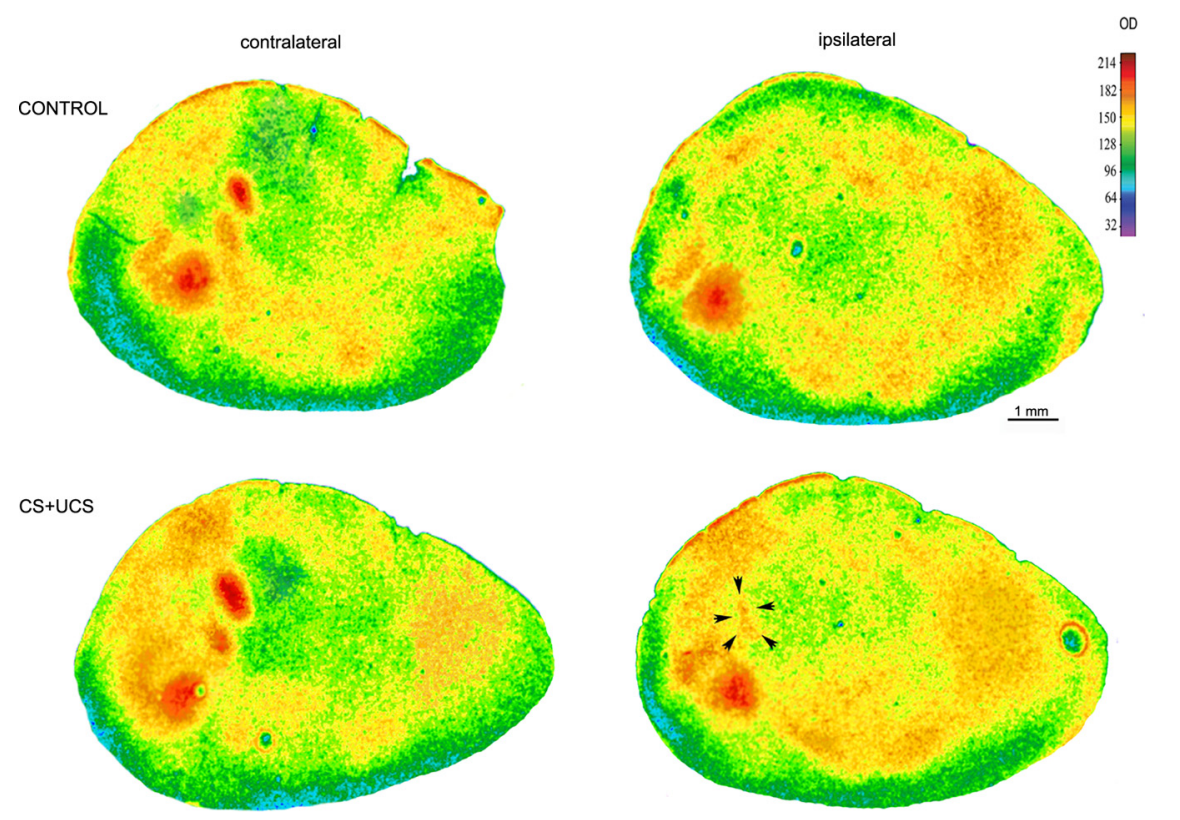

Figure 4. 2DG autoradiograms showing the effect of unilateral stimulation of row B vibrissae in control and CS + UCS mice on functional activation of primary and secondary somatosensory cortices. In control mice, only unilateral activation of SI and SII can be seen. In the ipsilateral hemisphere of trained animals, no activation in the barrel field is present. In SII, a distinct patch of activation appeared (arrowheads). In autoradiograms from trained mice, labeling in contralateral SI and SII is wider.
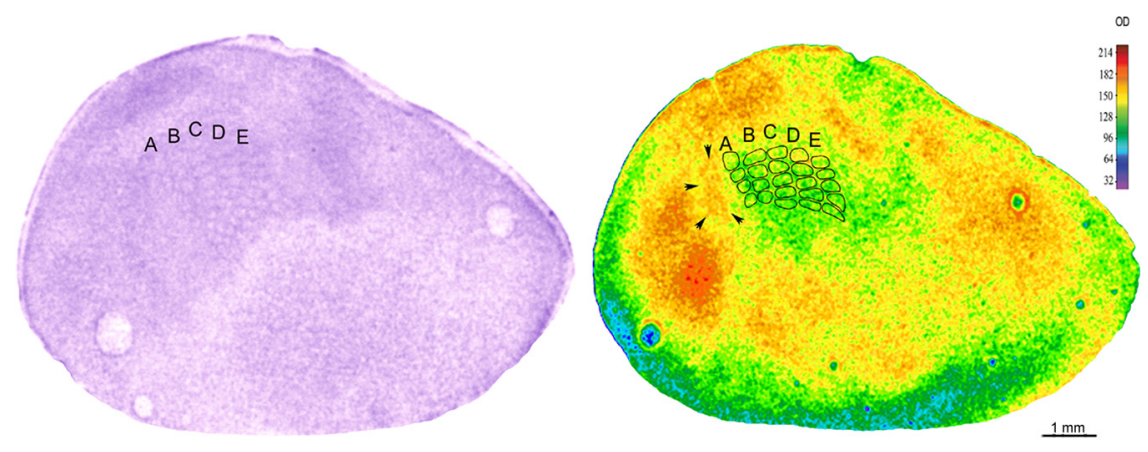

Figure 5. Tangential sections in the level of cortical layer IV of ipsilateral hemisphere of trained mouse. Left, Nissl staining showing localization and topography of barrel field. Right, Autoradiogram presenting the patch of 2DG labeling (arrowheads) and its localization in relation to the barrel field. A-E, Rows of barrels.

to the trained vibrissae. The response to ipsilateral vibrissae stimulation was first described by Pidoux and Verley (1979) in rats and mice. They described the ipsilateral potentials as having higher thresholds, smaller amplitudes, and longer latencies than contralateral potentials. They also showed that removing or cooling of contralateral barrel field or section of the corpus callosum eliminated them, proving their callosal origin. Interhemispheric connections between the barrel fields were described by Olavarria et al. (1984) and Koralek et al. (1990) in the rat, who found that they are confined to the septal regions. The work on unanesthetized rats by Shuler et al. (2001) and Wiest et al. (2010) found a proportion of units that responded to stimulation of vibrissae from either side of the muzzle in layer $\mathrm{V}$ of the barrel cortex. Bilateral subthreshold receptive fields were also described in that layer by Manns et al. (2004). However, in many 2DG investigations, ipsilateral labeling could not be found after stimulation of single vibrissae (Melzer et al., 1985; Chmielowska et al., 1986; Kossut et al., 1988; Sharp et al., 1988). Stimulation of all whiskers, however, brought some (although not quite significant) accumulation of 2DG uptake in the ipsilateral barrel field (Cybulska-
Klosowicz et al., 2006). Apparently, the ipsilateral functional activation of the barrel field is not strong enough to result in significant accumulation of label. Here, we confirmed the previous data in the situation when only one row of whiskers was stimulated, which resulted in no labeling discernible from background either in ipsilateral posteromedial barrel subfield or in ipsilateral SII (Fig. 4).

However, in the mice that underwent the conditioning, unilateral stimulation of the trained row of vibrissae produced labeling in ipsilateral SII (but not in SI). Therefore, our results point to the bilaterally integrative activity of SII under the influence of attentional/modulatory factors operating during learning. One of them is the modulatory influence of the tail shock (UCS), which is probably bilateral and can presumably affect the neuronal processing of input from the stimulated vibrissae in both hemispheres. In humans, pain activates SII, which is regarded as participating in distinguishing painful stimulations (Wang et al., 2010). In rats, painful electrical stimulation of feet or tail is associated with increased activity of catecholaminergic and serotonergic neurons in the brain (Sara, 2009) and release of neuromodulators essential for plasticity (Li et al., 2006). The locus ceruleus is activated by task-related stimuli in cognitive task (Aston-Jones et al., 1996), and the activation is closely correlated with pavlovian behavior (Bouret and Richmond, 2009). Both the locus ceruleus and the dorsal raphe send direct projections to the barrel cortex and VPM/VPL (Lee et al., 2008,2009 ) and can influence plasticity of vibrissal representations.

We propose that SI plasticity is primarily driven by a strong sensory input, and thus lateralized after unilateral stimulation during conditioning, whereas SII plasticity may be more dependent on neuromodulatory and top-down attentional factors, and thus bilateral. We have demonstrated previously (Cybulska-Klosowicz et al., 2009) that, during conditioning in the paradigm used in the present experiment, all thalamic somatosensory nuclei, the amygdala, nucleus basalis, and association cortical areas were activated equally strongly in both hemispheres. Moreover, the importance of associative processes and not just painful tail stimulation is stressed by the fact that no remodeling of cortical representation in either SI or SII was observed in the pseudoconditioned group. After pseudoconditioning, intensity of 2DG uptake in SII increased compared with controls, although significantly less than in trained animals; it may be supposed that the change in reactivity was not strong enough to result in an increase of cortical representation.

Interestingly, it appears that cognitive influences/attentional effects are more frequent, more robust, and more complex in SII, compared with SI (Meftah et al., 2002). There are at least two independent processes at the cortical level that are activated by 
selective attention in primates (Chapman and Meftah, 2005); additive effect, seen in SI and SII cells, observed during tactile texture discrimination tasks and multiplicative effect of selective attention (increase in response gain) localized to SII. Human brain imaging studies show that bilateral SII activation during tactile stimulation depends on the level of attention (Fujiwara et al., 2002). Our observations correspond well with findings in primates, in which bilateral activation of SII during attentional tasks is stronger (Burton et al., 1999).

Human studies show that somatosensory stimulation in tasks requiring increased attention to stimuli activate contralateral SI and bilaterally SII (Johansen-Berg et al., 2000; Chen et al., 2010). In the conditioning task that we applied, it is difficult to estimate the attentional component. Our previous work found increased interhemispheric interactions at the beginning of conditioning, but not on the last day of training in the conditioned, but not in the pseudoconditioned group (Cybulska-Klosowicz and Kossut, 2006). It was ascribed to increased arousal, which was high at the beginning of the training in the conditioned animals and remained high throughout its duration in the pseudoconditioned ones. Therefore, we can probably eliminate arousal as a factor that induces plasticity in SII.

We demonstrated that a simple form of associative learning, classical conditioning of unilateral somatosensory input, produces bilateral plastic changes in responsiveness of the second somatosensory representation. We propose that it is attributable to integrative role of SII, its lesser topographical specificity, and strong involvement in attentional processing and learning.

\section{References}

Adrian ED (1941) Afferent discharges to the cerebral cortex from peripheral sense organs. J Physiol 100:159-191.

Aston-Jones G, Rajkowski J, Kubiak P, Valentino RJ, Shipley MT (1996) Role of the locus coeruleus in emotional activation. Prog Brain Res 107:379-402.

Bohlhalter S, Fretz C, Weder B (2002) Hierarchical versus parallel processing in tactile object recognition: a behavioural-neuroanatomical study of aperceptive tactile agnosia. Brain 125:2537-2548.

Bouret S, Richmond BJ (2009) Relation of locus coeruleus neurons in monkeys to pavlovian and operant behaviors. J Neurophysiol 101:898-911.

Brett-Green B, Fifková E, Larue DT, Winer JA, Barth DS (2003) A multisensory zone in rat parietotemporal cortex: intra- and extracellular physiology and thalamocortical connections. J Comp Neurol 460:223-237.

Burton H, Carlson M (1986) Second somatic sensory cortical area (SII) in a prosimian primate, Galago crassicaudatus. J Comp Neurol 247:200-220.

Burton H, Kopf EM (1984) Connections between the thalamus and the somatosensory areas of the anterior ectosylvian gyrus in the cat. J Comp Neurol 224:173-205.

Burton H, Abend NS, MacLeod AM, Sinclair RJ, Snyder AZ, Raichle ME (1999) Tactile attention tasks enhance activation in somatosensory regions of parietal cortex: a positron emission tomography study. Cereb Cortex 9:662-674.

Carvell GE, Simons DJ (1986) Somatotopic organization of the second somatosensory area (SII) in the cerebral cortex of the mouse. Somatosens Res 3:213-237.

Carvell GE, Simons DJ (1987) Thalamic and corticocortical connections of the second somatic sensory area of the mouse. J Comp Neurol 265:409-427.

Chapman CE, Meftah el-M (2005) Independent controls of attentional influences in primary and secondary somatosensory cortex. J Neurophysiol 94:4094-4107.

Chen TL, Babiloni C, Ferretti A, Perrucci MG, Romani GL, Rossini PM, Tartaro A, Del Gratta C (2010) Effects of somatosensory stimulation and attention on human somatosensory cortex: an fMRI study. Neuroimage 53:181-188.

Chmielowska J, Kossut M, Chmielowski M (1986) Single vibrissal cortical column in the mouse labeled with 2-deoxyglucose. Exp Brain Res 63:607-619.
Cybulska-Klosowicz A, Kossut M (2006) Early-phase of learning enhances communication between brain hemispheres. Eur J Neurosci 24:1470-1476.

Cybulska-Klosowicz A, Zakrzewska R, Kossut M (2009) Brain activation patterns during classical conditioning with appetitive or aversive UCS. Behav Brain Res 204:102-111.

Fanselow MS, Poulos AM (2005) The neuroscience of mammalian associative learning. Annu Rev Psychol 56:207-234.

Fujiwara N, Imai M, Nagamine T, Mima T, Oga T, Takeshita K, Toma K, Shibasaki H (2002) Second somatosensory area (SII) plays a significant role in selective somatosensory attention. Brain Res Cogn Brain Res 14:389-397.

Iwamura Y (2000) Bilateral receptive field neurons and callosal connections in the somatosensory cortex. Philos Trans R Soc Lond B Biol Sci 355:267-273.

Jasinska M, Siucinska E, Cybulska-Klosowicz A, Pyza E, Furness DN, Kossut M, Glazewski S (2010) Rapid, learning-induced inhibitory synaptogenesis in murine barrel field. J Neurosci 30:1176-1184.

Johansen-Berg H, Christensen V, Woolrich M, Matthews PM (2000) Attention to touch modulates activity in both primary and secondary somatosensory areas. Neuroreport 11:1237-1241.

Koralek KA, Olavarria J, Killackey HP (1990) Areal and laminar organization of corticocortical projections in the rat somatosensory cortex. J Comp Neurol 299:133-150.

Kossut M, Hand PJ, Greenberg J, Hand CL (1988) Single vibrissal cortical column in SI cortex of rat and its alterations in neonatal and adult vibrissa-deafferented animals: a quantitative 2DG study. J Neurophysiol 60:829-852.

Kostopoulos P, Albanese MC, Petrides M (2007) Ventrolateral prefrontal cortex and tactile memory disambiguation in the human brain. Proc Natl Acad Sci U S A 104:10223-10228.

Kwegyir-Afful EE, Keller A (2004) Response properties of whiskerrelated neurons in rat second somatosensory cortex. J Neurophysiol 92:2083-2092.

Lee SB, Lee HS, Waterhouse BD (2008) The collateral projection from the dorsal raphe nucleus to whisker-related, trigeminal sensory and facial motor systems in the rat. Brain Res 1214:11-22.

Lee SB, Beak SK, Park SH, Waterhouse BD, Lee HS (2009) Collateral projection from the locus coeruleus to whisker-related sensory and motor brain regions of the rat. J Comp Neurol 514:387-402.

Li L, Ebner FF (2006) Balancing bilateral sensory activity: callosal processing modulates sensory transmission through the contralateral thalamus by altering the response threshold. Exp Brain Res 172:397-415.

Li SC, Brehmer Y, Shing YL, Werkle-Bergner M, Lindenberger U (2006) Neuromodulation of associative and organizational plasticity across the life span: empirical evidence and neurocomputational modeling. Neurosci Biobehav Rev 30:775-790.

Liao CC, Yen CT (2008) Functional connectivity of the secondary somatosensory cortex of the rat. Anat Rec (Hoboken) 291:960-973.

Manns ID, Sakmann B, Brecht M (2004) Sub- and suprathreshold receptive field properties of pyramidal neurones in layers $5 \mathrm{~A}$ and $5 \mathrm{~B}$ of rat somatosensory barrel cortex. J Physiol 556:601-622.

Manzoni T, Caminiti R, Spidalieri G, Morelli E (1979) Anatomical and functional aspects of the associative projections from somatic area SI to SII. Exp Brain Res 34:453-470.

Meftah el-M, Shenasa J, Chapman CE (2002) Effects of a cross-modal manipulation of attention on somatosensory cortical neuronal responses to tactile stimuli in the monkey. J Neurophysiol 88:3133-3149.

Melzer P, Van der Loos H, Dörfl J, Welker E, Robert P, Emery D, Berrini JC (1985) A magnetic device to stimulate selected whiskers of freely moving or restrained small rodents: its application in a deoxyglucose study. Brain Res 348:229-240.

Miyashita E, Keller A, Asanuma H (1994) Input-output organization of the rat vibrissal motor cortex. Exp Brain Res 99:223-232.

Olavarria J, Van Sluyters RC, Killackey HP (1984) Evidence for the complementary organization of callosal and thalamic connections within rat somatosensory cortex. Brain Res 291:364-368.

Pidoux B, Verley R (1979) Projections on the cortical somatic I barrel subfield from ipsilateral vibrissae in adult rodents. Electroencephalogr Clin Neurophysiol 46:715-726.

Pleger B, Foerster AF, Ragert P, Dinse HR, Schwenkreis P, Malin JP, Nicolas V, 
Tegenthoff M (2003) Functional imaging of perceptual learning in human primary and secondary somatosensory cortex. Neuron 40:643-653.

Pons TP, Garraghty PE, Mishkin M (1988) Lesion-induced plasticity in the second somatosensory cortex of adult macaques. Proc Natl Acad Sci U S A 85:5279-5281.

Quallo MM, Price CJ, Ueno K, Asamizuya T, Cheng K, Lemon RN, Iriki A (2009) Gray and white matter changes associated with tool-use learning in macaque monkeys. Proc Natl Acad Sci U S A 106:18379-18384.

Raij T, Karhu J, Kicić D, Lioumis P, Julkunen P, Lin FH, Ahveninen J, Ilmoniemi RJ, Mäkelä JP, Hämäläinen M, Rosen BR, Belliveau JW (2008) Parallel input makes the brain run faster. Neuroimage 40:1792-1797.

Rizzolatti G, Luppino G (2001) The cortical motor system. Neuron 31:889-901.

Sacco T, Sacchetti B (2010) Role of secondary sensory cortices in emotional memory storage and retrieval in rats. Science 329:649-656.

Sara SJ (2009) The locus coeruleus and noradrenergic modulation of cognition. Nat Rev Neurosci 10:211-223.

Sharp FR, Gonzalez MF, Morgan CW, Morton MT, Sharp JW (1988) Common fur and mystacial vibrissae parallel sensory pathways: ${ }^{14} \mathrm{C}$ 2-deoxyglucose and WGA-HRP studies in the rat. J Comp Neurol 270:446-469.
Shi CJ, Cassell MD (1998) Cascade projections from somatosensory cortex to the rat basolateral amygdala via the parietal insular cortex. J Comp Neurol 399:469-491.

Shuler MG, Krupa DJ, Nicolelis MA (2001) Bilateral integration of whisker information in the primary somatosensory cortex of rats. J Neurosci 21:5251-5261.

Siucinska E, Kossut M (1996) Short-lasting classical conditioning induces reversible changes of representational maps of vibrissae in mouse SI cortex-a 2DG study. Cereb Cortex 6:506-513.

Theyel BB, Llano DA, Sherman SM (2010) The corticothalamocortical circuit drives higher-order cortex in the mouse. Nat Neurosci 13:84-88.

Wang W, Zhang M, Wang Y, Jin C, Yan B, Ma S (2010) 5-HT modulation of pain in SI and SII revealed by fMRI. Zhong Nan Da Xue Xue Bao Yi Xue Ban 35:185-193.

Wiest MC, Thomson E, Pantoja J, Nicolelis MA (2010) Changes in S1 neural responses during tactile discrimination learning. J Neurophysiol 104:300-312.

Woolsey TA (1967) Somatosensory, auditory and visual cortical areas of the mouse. Johns Hopkins Med J 121:91-112. 